\title{
Effects of rifampicin on osteogenic differentiation and proliferation of human mesenchymal stem cells in the bone marrow
}

\author{
Z. Zhang, X. Wang, F. Luo, H. Yang, T. Hou, Q. Zhou, F. Dai, Q. He and J. Xu \\ Department of Orthopaedics, Southwest Hospital, \\ Third Military Medical University, ChongQing, China \\ Corresponding author: J. Xu \\ E-mail: xjzslw@163.com
}

Genet. Mol. Res. 13 (3): 6398-6410 (2014)

Received February 4, 2013

Accepted July 26, 2013

Published August 25, 2014

DOI http://dx.doi.org/10.4238/2014.August.25.3

\begin{abstract}
This study was designed to investigate the effect of different concentrations of rifampicin on osteogenic differentiation and proliferation of mesenchymal stem cells (MSCs) in human bone marrow. Rifampicin treatment at $0,4,8,16,32,64$, and $128 \mu \mathrm{g} / \mathrm{mL}$ was applied throughout the whole process, from stromal cells purified from human bone marrow to differentiated bone cells. The effect of rifampicin on MSC proliferation was determined using the MTT assay. The effect of rifampicin on the expressions of type I collagen (COL1A1), osteopontin/ bone Gla protein (OPN/BGP), and alkaline phosphatase (ALP) in human osteoblast cells were determined by real-time polymerase chain reaction, and the expressions of COL1A1, OPN/BGP, and the runt-related transcription factor (RUNX2) were determined by Western blot. Results showed that the proliferation of MSCs was significantly inhibited when the rifampicin concentration exceeded $32 \mu \mathrm{g} / \mathrm{mL}$. In addition, increased rifampicin concentrations inhibited the formation of calcium nodules, OPN/BGP, and COL1A1 in osteoblasts after 28 days of induction. The RNA expressions of OPN/BGP, COL1A1, and ALP were significantly downregulated compared to those of the control group in osteoblasts
\end{abstract}


after induction. The protein expressions of RUNX2, COL1A1, and OPN/BGP were also significantly downregulated compared to those of the control group after induction. In conclusion, rifampicin at exorbitant concentration exerts adverse effects on the proliferation of MSCs in human bone marrow and the differentiation of osteoblasts.

Key words: Rifampicin; Proliferation; Mesenchymal stem cell; Osteoblast differentiation

\section{INTRODUCTION}

Rifampicin is a potent antibiotic that is especially effective against Staphylococcus, which sometimes shows resistance to beta-lactam antibiotics (O'Reilly et al., 1992; Littlewood-Evans et al., 1997). The efficiency of the combination therapy of rifampicin and ciprofloxacin in controlling orthopedic infections has been demonstrated in clinical and experimental studies (Hendry et al., 1987; Dworkin et al., 1990).

Several studies shown that after oral administration of rifampicin, the rifampicin concentration in bone and in the joint tuberculosis focus was lower than the effective bactericidal concentration (Roth, 1984; Widmer et al., 1992; Mahajan et al., 1997; Zimmerli et al., 1998; Isefuku et al., 2001). Therefore, the topical application of rifampicin on tuberculosis foci has been considered as an approach worth investigating. Exorbitant concentrations of rifampicin have adverse impacts on bone formation. Therefore, in clinic applications, it is necessary to achieve a balance between anti-tuberculosis therapy and bone formation protection. Osteoblasts derived from mesenchymal stem cells (MSCs) in bone marrow are the main functional cells in bone formation and are responsible for the synthesis, secretion, and mineralization of the bone matrix. The specific biomarkers include type I collagen (COL1A1) and the osteopontin/bone Gla protein (OPN/BGP). In this study, different rifampicin concentrations were applied throughout the entire bone formation process, from stromal cells purified from human bone marrow to differentiated bone cells. The effect of rifampicin on MSC proliferation was determined using the 3-4(4,5-dimethylthiazol2-yl)-2,5-diphenyltetrazolium bromide (MTT) assay. The effect of rifampicin on the expressions of COL1A1, OPN/BGP, and alkaline phosphatase (ALP) in human osteoblasts were determined by real-time reverse transcription-polymerase chain reaction (RT-PCR), and the protein expressions of RUNX2, COL1A1, and OPN/BGP were determined by Western blot methods. These results should provide experimental data for the amendment of delayed release parameters, clinical doses, and course of treatment of rifampicin to further investigate the effect of rifampicin on osteogenic differentiation and the proliferation of MSCs in human bone marrow.

\section{MATERIAL AND METHODS}

\section{Culture and identification of human MSCs}

\section{Isolation and culture of human primary MSCs}

Three volunteers who underwent spinal posterior fusion operations were selected as described previously (Costerton et al., 1999). After the operation, the posterior iliac crests of the 
patients were punctured and $40 \mathrm{~mL}$ bone marrow was drawn with a heparin pre-wet syringe, of which $30 \mathrm{~mL}$ was mixed with demineralized bone matrix for confluence and $10 \mathrm{~mL}$ was used in the experiment. The bone marrow was slowly added to the centrifuge tube with lymphocyte separation medium containing an equal volume of $1.077 \mathrm{~g} / \mathrm{mL}$ Percol, and the boundary between them was clear. After centrifuging at $3000 \mathrm{rpm}$ for $20 \mathrm{~min}$, the white, cloudy middle layer of nucleated cells was drawn, $10 \mathrm{~mL} \mathrm{D}$-Hanks solution was added, and the solution was repeatedly pipetted and rinsed. After centrifuging at $1500 \mathrm{rpm}$ for $6 \mathrm{~min}$, the supernatant was removed. The precipitation was added with $2 \mathrm{~mL}$ primary culture medium [Dulbecco's modified Eagle's medium (DMEM)/ F12, containing $15 \%$ fetal calf serum], and was repeatedly pipetted into single cell suspension. After the cells were counted, $5 \times 10^{5}$ cells $/ \mathrm{cm}^{2}$ were inoculated in flasks and placed in a $37^{\circ} \mathrm{C}, 5 \%$ $\mathrm{CO}_{2}$ humidified incubation chamber. Half of the medium was changed after $24 \mathrm{~h}$, and the medium was changed completely after 4 days, at which point the suspended cells were abandoned. The medium was changed every two to three days thereafter. Cell subculture was performed once the cells were fused into single cells and approximately $80 \%$ of cells covered the bottom.

\section{Subculture of human MSCs}

The primary culture medium was discarded in the bottle, and $2 \mathrm{~mL} 0.25 \%$ trypsin digestion was added and shaken to allow the digestive juices to flow through the culture bottle completely. The culture medium in the bottle was then removed. The digestive juices were discarded, and $2 \mathrm{~mL}$ digestive juice was again added to digest for $1-3 \mathrm{~min}$ at $37^{\circ} \mathrm{C}$. Cells were observed under an inverted microscope large gap. The intercellular space was widened, and the cells were shrunk and turned around. After the partial cells fell off, the same amount of mass cell culture medium was added to terminate the digestion. Adherent cells all fell off after gentle pipetting with a straw, and the scattered cell clusters were collected in a centrifuge tube. After centrifugation at $1000 \mathrm{rpm}$ for $6 \mathrm{~min}$, the supernatant was discarded, and D-Hanks solution was added. After rinsing, suitable passage cell culture medium was added, and the cells were completely pipetted into a single cell suspension. After the harvested cells were counted, $1 \times 10^{4}$ cells $/ \mathrm{cm}^{2}$ were inoculated in flasks, $10 \mathrm{~mL}$ passage cell culture medium was added, and they were incubated at $37^{\circ} \mathrm{C}$ and $5 \% \mathrm{CO}_{2}$ in a humidified incubation chamber. The culture medium was replaced every other day, and cell growth conditions and morphological changes were observed daily under an inverted microscope.

\section{Determination of cell viability}

Single cell suspensions were prepared according to the cell digestion method. Nine drops cell suspension and one drop $0.4 \%$ Trypan blue solution were added into small test tubes respectively, and mixed well. After $3 \mathrm{~min}$, the living and dead cells were counted in a blood cell counting chamber. Cell viability was determined according to the following formula: rate of living cells $=$ total number of living cells/total number of living cells + total number of dead cells.

\section{Effect of rifampicin concentration on the proliferation of human MSCs}

\section{Observations under inverted microscope}

The third generation MSCs were collected and inoculated at a density of $1 \times 10^{4}$ cells $/ \mathrm{mL}$ 
on 24-well plates. According to the concentration of added rifampicin, the cells were divided into seven experimental groups treated with $0,4,8,16,32,64$, and $128 \mu \mathrm{g} / \mathrm{mL}$ rifampicin, termed as groups 1 to 7 , respectively. The control group (group 1) was treated with only vehicle. As of seven days after inoculation, the growth of MSCs was observed under the microscope daily.

\section{MTT cell proliferation assay}

Each group was set a blank control in the MTT assay, which was treated with the same concentration of rifampicin $(4,8,16,32,64$, or $128 \mu \mathrm{g} / \mathrm{mL})$. The MTT assay was performed on the first day. In the experimental groups, $4,8,16,32,64$, or $128 \mu \mathrm{g} / \mathrm{mL}$ rifampicin was added into each well, and three wells were used as blank controls. On the third, fifth, and seventh days, three wells were chosen for the MTT test. According to the MTT assay protocol, $490 \mathrm{~nm}$ was selected to test the optical density (OD) value in the microplate reader. Time was used as the horizontal axis, and the OD value (mean of three wells) was used as the longitudinal axis to draw the cell growth curve in each group.

\section{Effects of rifampicin concentration on the differentiation of human MSCs}

\section{Alizarin red staining of calcium nodules}

The third generation MSCs were obtained, seeded on a 6-well plate with pre-culture coverslips, and induction culture medium was added for culture. According to the concentration of rifampicin, the cells were divided into seven groups as described above. After seven days of culture, the growth of MSCs and the formation of round or oval nodules were observed daily under the microscope. Twenty-eight days after the induction, the coverslip was removed from each group, cells were washed three times with phosphate-buffered saline (PBS), fixed in $75 \%$ alcohol for $30 \mathrm{~min}$, and stained with alizarin red dye for 3-5 min. After dehydration of the gradient alcohol xylene, the sections were mounted with neutral resin.

\section{Immunohistochemical staining of osteocalcin and type I collagen}

The third generation MSCs were obtained, seeded on 6-well plates with pre-culture coverslips, and induction culture medium was added for culture. According to the concentration of rifampicin, the cells were divided into seven groups as described above. After 28 days of culture, the coverslip was removed, and cells were washed three times with PBS. The sections were fixed with $95 \%$ alcohol for 20 min according to the SABC immunohistochemical staining protocol.

\section{Real-time RT-PCR}

The cells were induced for 28 days, and cells in each experimental group were collected. The cells cultured in media without rifampicin osteogenic induction were used as controls. In accordance with the instructions of the RNeasy Mini Kit (Qiagen), the corresponding RNA of cells were obtained by schizolysis, extraction, and purification, and then RNase-free DNase I (Qiagen) was used to remove any genomic DNA in the cells. RNA concentrations were measured using a spectrophotometer. Real-time RT-PCR was performed in accordance with the SYBR ${ }^{\circledR}$ ExScript $^{\mathrm{TM}}$ 
RT-PCR Kit (Perfect Real Time, TaKaRa) two-step instructions. The specific protocol was as follows. The $20-\mu \mathrm{L}$ reverse transcription reaction system included $1 \mu \mathrm{g}$ RNA and corresponding reagents. Under the action of M-MLV reverse transcriptase, the program was set to $42^{\circ} \mathrm{C}$ for $15 \mathrm{~min}$ and $92^{\circ} \mathrm{C}$ for $2 \mathrm{~min}$. The $20-\mu \mathrm{L}$ PCR system included $1 \mu \mathrm{L}$ cDNA and corresponding reagents, and the program was set to $95^{\circ} \mathrm{C}$ for $30 \mathrm{~s}, 95^{\circ} \mathrm{C}$ for $15 \mathrm{~s}, 54^{\circ} \mathrm{C}$ for $10 \mathrm{~s}$, and $72^{\circ} \mathrm{C}$ for $10 \mathrm{~s}(40$ cycles); the melting curve ranged from 65 to $95^{\circ} \mathrm{C}$, and the board was read once every $1{ }^{\circ} \mathrm{C}$. COL1A1, OPN/BGP, and ALP expressions were detected, and $18 \mathrm{~S}$ was used as a housekeeping gene for the relative quantification of gene expression (gene specific sequence of Table 1). The standard curve was prepared based on the cDNA of human osteoblasts and set to 50,10, 2, 0.4, 0.08, and $0.016 \mathrm{ng}$.

Table 1. Real-time RT-PCR primers.
\begin{tabular}{llr} 
& \\
\hline Target gene & Oligonucleotide sequence & Expected product (bp) \\
\hline 18 S forward & 5'-GTAACCCGTTGAACCCCATT-3' & 151 \\
18 S reverse & 5'-CCATCCAATCGGTAGTAGCG-3' \\
ALP forward & 5'-CCCACAATGTGGACTACCT-3' & 143 \\
ALP reverse & 5'-GAAGCCTTTGGGGTTCTTC-3' \\
OPN forward & 5'-TTCCAAGTAAGTCCAACGAAAG-3' \\
OPN reverse & 5'-GTGACCAGTTCATCAGATTCAT-3' & 181 \\
COL 1A1 forward & 5'-TCCAACGAGATCGAGATCC-3' \\
COL 1A1 reverse & 5'-AAGCCGAATTCCTGGTCT-3' & 195 \\
\hline
\end{tabular}

\section{Western blot}

The cells were induced for 28 days, and cells in each experimental group were respectively collected. The cells cultured in media without rifampicin osteogenic induction were used as controls. Cell lysis was performed and total soluble protein was extracted according to RIPA cell lysate kit instructions. The cells were washed three times with PBS, and $0.2 \mathrm{~mL}$ lysis buffer was used for lysing cells in $100 \mathrm{~mL}$ flasks (phenylmethanesulfonyl fluoride was added before use), and placed on ice for $30 \mathrm{~min}$. The cells on both sides of the bottle were scraped and the lysate was collected. After centrifugation at $4^{\circ} \mathrm{C}$ at $20,000 \mathrm{rpm}$ for $20 \mathrm{~min}$, the supernatant protein was collected and packed into small portions at $-20^{\circ} \mathrm{C}$ after the concentration was determined.

The runt-related transcription factor (RUNX2) was obtained on 12, 8, and 15\% separating gels, respectively, and COL1AI, OPN/BGP, and the internal reference $\beta$-actin (purchased from Abcam) proteins were obtained on $12 \%$ separating gels. The proteins were transferred to the nitrocellulose membrane under $80 \mathrm{~V}$ regulator, terminated with 5\% skim milk Tris-buffered saline with Tween 20 (TBS-T) solution for $2 \mathrm{~h}$, and incubated with the appropriate primary antibodies for RUNX2 (Santa Cruz, rabbit polyclonal antibody, 1:1000), collagen I (Santa Cruz, goat polyclonal antibody, 1:1000), osteocalcin (Abcam, mouse monoclonal antibody, 1: 500), and $\beta$-actin (Abcam, mouse monoclonal antibody, 1:5000) at $4^{\circ} \mathrm{C}$ overnight. Proteins were then incubated for a further $1 \mathrm{~h}$ in the secondary antibody (horseradish peroxidase, Southern Biotech 1:1000-1:2000). After rinsing, images were obtained with the ECL enhanced chemiluminescence kit (KPL Inc.), and captured images were scanned and analyzed with a gel imaging system.

\section{Statistical analysis}

The data are reported as means \pm standard deviation. Single-factor analysis of variance 
(ANOVA) and least significant difference (LSD) statistical methods were used for comparisons between groups based on the SPSS 13.0 software; $\mathrm{P}<0.05$ indicated a significant difference.

\section{RESULTS}

\section{Cellular morphology observations under inverted microscope}

Growth and morphological characteristics of cells in each group were observed daily under inverted microscope. The cells in all experimental groups began to adhere, spread, and restore to spindle-shape after 2-4 h. Eight to $10 \mathrm{~h}$ after adherence of living cells, the cells in each group were polygon and scale-shaped. After $24 \mathrm{~h}$, the cells in all experimental groups were observed to begin to proliferate, although the proliferation rate was significantly slower than that of cells cultured using routine methods. Three days later, the morphology of cells was polygonal, and the volume of cells was slightly increased. The cells showed aggregation growth, increased secretion of extracellular matrix, Trypan blue staining, and the cell viability increased to $99 \%$. Seven days later, clear round or oval nodules appeared, a high refraction of vacuoles and dark granules were secreted, and the cells around nodules showed radial distribution. The cells in group 4 showed necrosis, and the cells in groups 5, 6, and 7 showed swelling, degeneration, and necrosis. There were significant differences in the morphology of cells in groups 4 to 7 compared with cells of groups 1,2, and 3 (Figure 1).
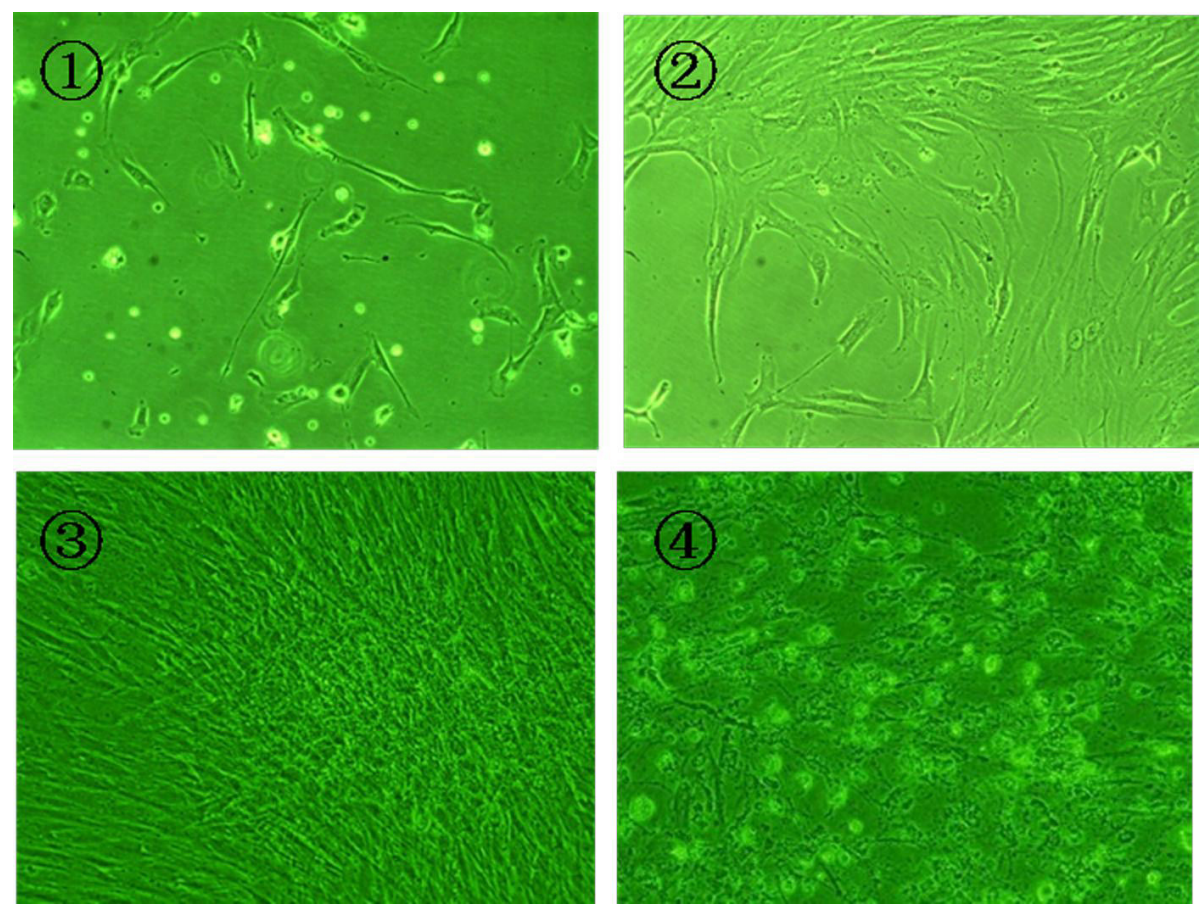

Figure 1. Growth condition and morphous characteristics of mesenchymal stem cell (MSCs) under inverted microscope. 1. Morphous of the third generation MSCs within $24 \mathrm{~h}$. 2. Morphous of MSCs in 3rd day of culture. 3. Morphous of MSCs in 7th day of culture. 4. Morphous of MSCs in 12th day of culture. 


\section{Effect of rifampicin on cell proliferation of MSCs}

MSCs were cultured in culture medium containing different concentrations of rifampicin, and OD values in the experimental and control groups were detected with the MTT assay after 1, 3, 5, and 7 days. The corresponding results (Table 2, Figure 2) showed that the proliferation of MSCs was significantly inhibited when the rifampicin concentration was higher than $32 \mu \mathrm{g} / \mathrm{mL}(\mathrm{P}<0.05)$. The cell proliferation in groups 5, 6, and 7 reached a peak on the fifth day and then declined thereafter, which showed that high rifampicin concentrations caused cell necrosis or apoptosis.

Table 2. Effects of rifampicin at diverse concentrations on cell proliferation determined by MTT.

\begin{tabular}{cccccccc}
\hline \multirow{2}{*}{ Time (days) } & \multicolumn{7}{c}{ Groups } \\
\cline { 2 - 8 } & 1 & 2 & 3 & 4 & 5 & 6 & 7 \\
\hline 1 & $0.277 \pm 0.011$ & $0.261 \pm 0.011$ & $0.254 \pm 0.012$ & $0.252 \pm 0.008$ & $0.248 \pm 0.016^{*}$ & $0.216 \pm 0.017^{*}$ & $0.144 \pm 0.019^{*}$ \\
3 & $0.528 \pm 0.011$ & $0.503 \pm 0.011$ & $0.496 \pm 0.012$ & $0.451 \pm 0.010$ & $0.420 \pm 0.018^{*}$ & $0.400 \pm 0.017^{*}$ & $0.275 \pm 0.020^{*}$ \\
5 & $0.860 \pm 0.007$ & $0.846 \pm 0.007$ & $0.843 \pm 0.010$ & $0.741 \pm 0.015$ & $0.723 \pm 0.019^{*}$ & $0.532 \pm 0.019^{*}$ & $0.421 \pm 0.015^{*}$ \\
7 & $0.886 \pm 0.011$ & $0.849 \pm 0.010$ & $0.859 \pm 0.019$ & $0.769 \pm 0.015$ & $0.602 \pm 0.015^{*}$ & $0.214 \pm 0.010^{*}$ & $0.195 \pm 0.016^{*}$ \\
\hline
\end{tabular}

*Compared with the control group (1), $\mathrm{P}<0.05$.

A
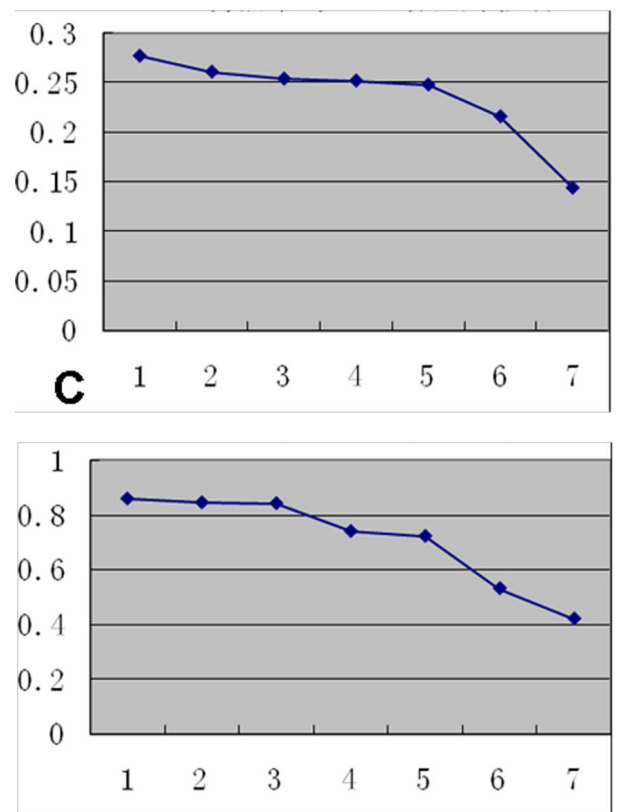

B
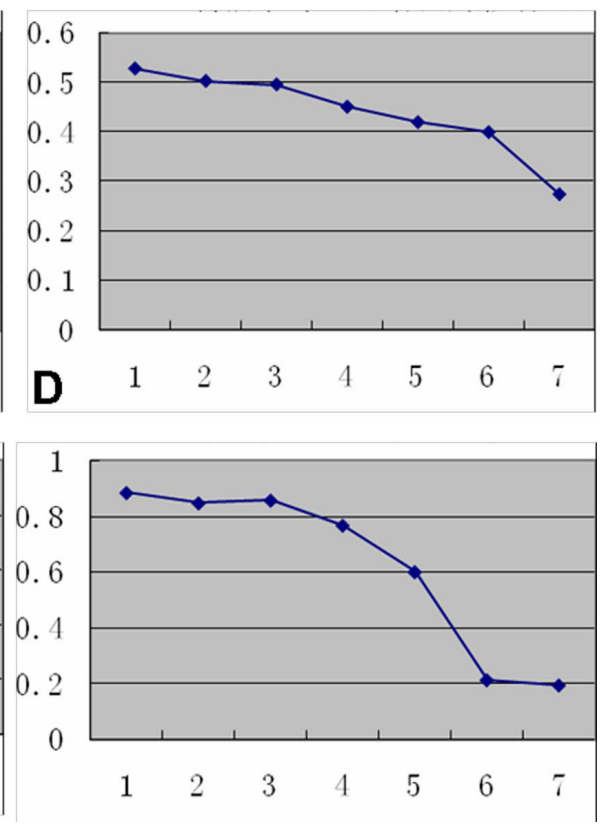

Figure 2. Effects of rifampicin at different concentrations on the proliferation of MSCs. A. Effects of rifampicine at different concentrations on the proliferation of MSCs on the 1st day. B. Effects of rifampicine at different concentrations on the proliferation of MSCs on the 3rd day. C. Effects of rifampicine at different concentrations on the proliferation of MSCs on the 5th day. D. Effects of rifampicine at different concentrations on the proliferation of MSCs on the 7th day. Numbers $1-7$ in long axis represent $0,4,8,16,32,64$, and $128 \mu \mathrm{g} / \mathrm{mL}$ rifampicin treatment group. 


\section{Morphology of MSCs after differentiation and alizarin red staining}

The calcium nodules in each experimental group were stained with alizarin red, which showed bright red bone-like tissue structures and a large amount of matrix and cells with red-stained nuclei were aggregated around its calcification centers. MSCs placed in the induction medium with different rifampicin concentrations showed obvious morphological changes. Most MSCs showed cord-like shapes, a few cells showed angular shapes, individual cells showed irregular shapes, and one to two nucleoli were observed in the nucleus. After induction, the cell volume increased, cells with polygonal and irregular shapes increased, and cytoplasmic particles were clear. Fourteen days later, some cells in groups 5, 6, and 7 groups showed necrosis and swelling. Alizarin red staining revealed the presence of mineralized calcium nodules in the cells. The generation of calcium nodules was inhibited with increasing rifampicin concentration (Figures 3 and 4).

\section{Effects of rifampicin on OCN and COL1 expression by immunohistochemical staining}

As shown in Figures 5 and 6, the expressions of OCN and COL1 in MSCs were significantly inhibited when the concentration of rifampicin was higher than $32 \mu \mathrm{g} / \mathrm{mL}$.

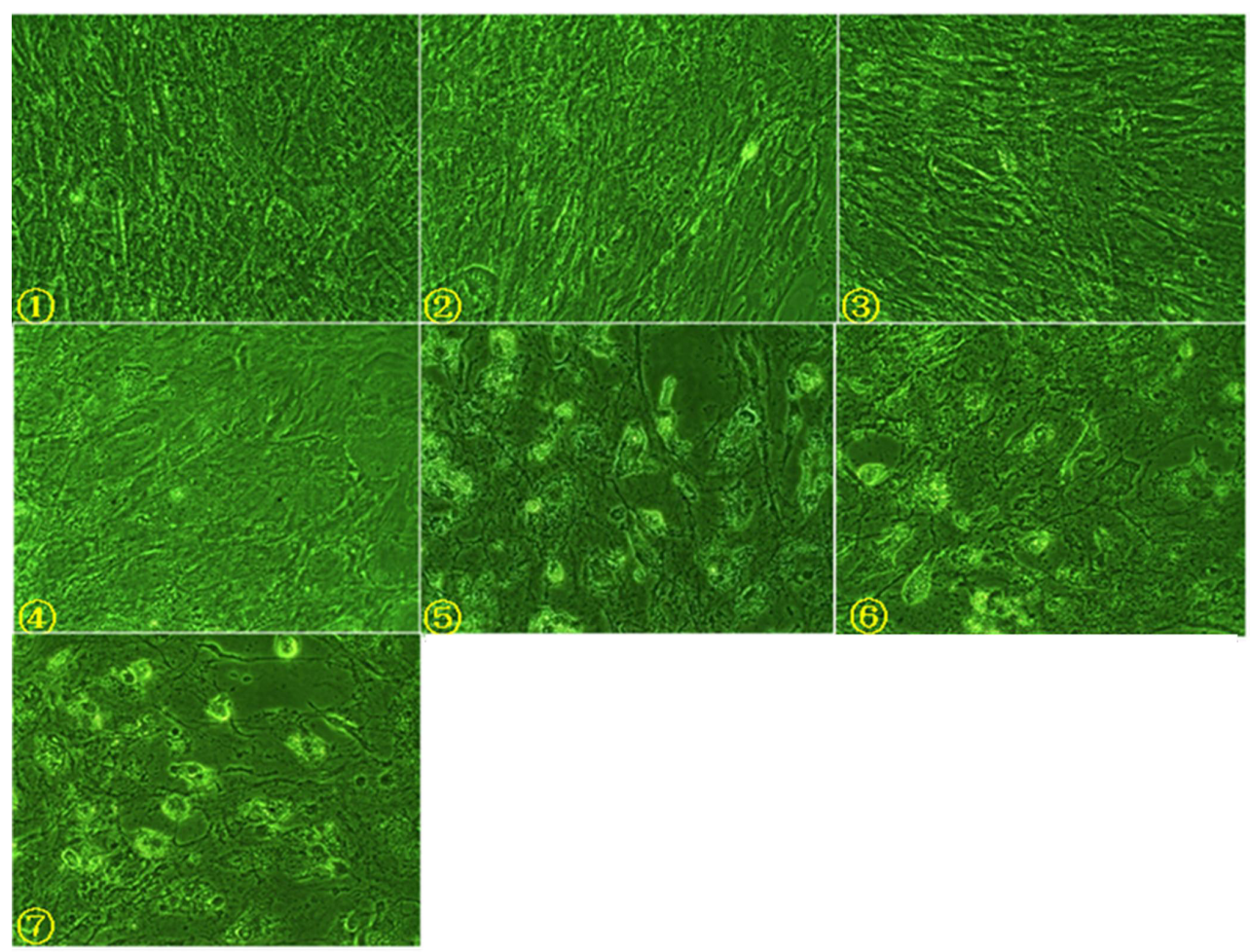

Figure 3. Effects of rifampicine at different concentrations on morphous of MSCs after 28 days of bone formation induction under inverted microscop. Numbers $1,2,3,4,5,6$, and 7 represent $0,4,8,16,32,64$, and $128 \mu \mathrm{g} / \mathrm{mL}$ rifampicin treatment group, respectively. 


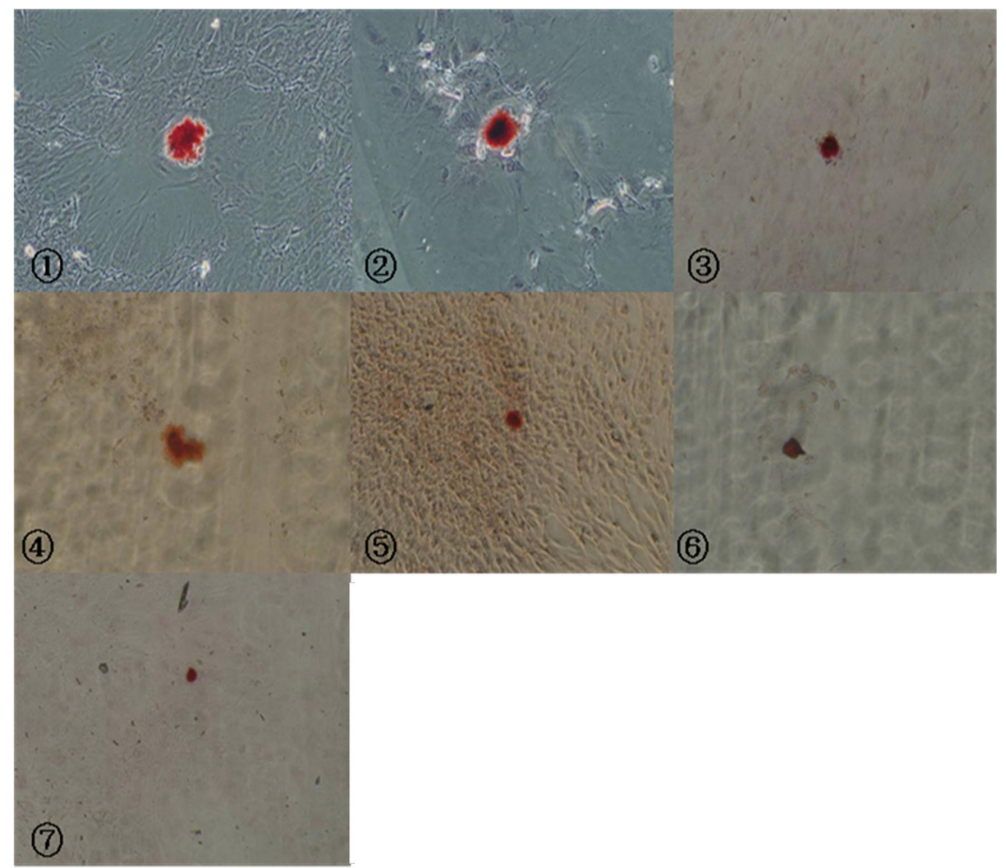

Figure 4. Effects of rifampicin at different concentrations on calcium nodules after 28 days of bone formation induction by alizarin red staining. Numbers $1,2,3,4,5,6$, and 7 represent $0,4,8,16,32,64$, and $128 \mu \mathrm{g} / \mathrm{mL}$ rifampicin treatment group, respectively.

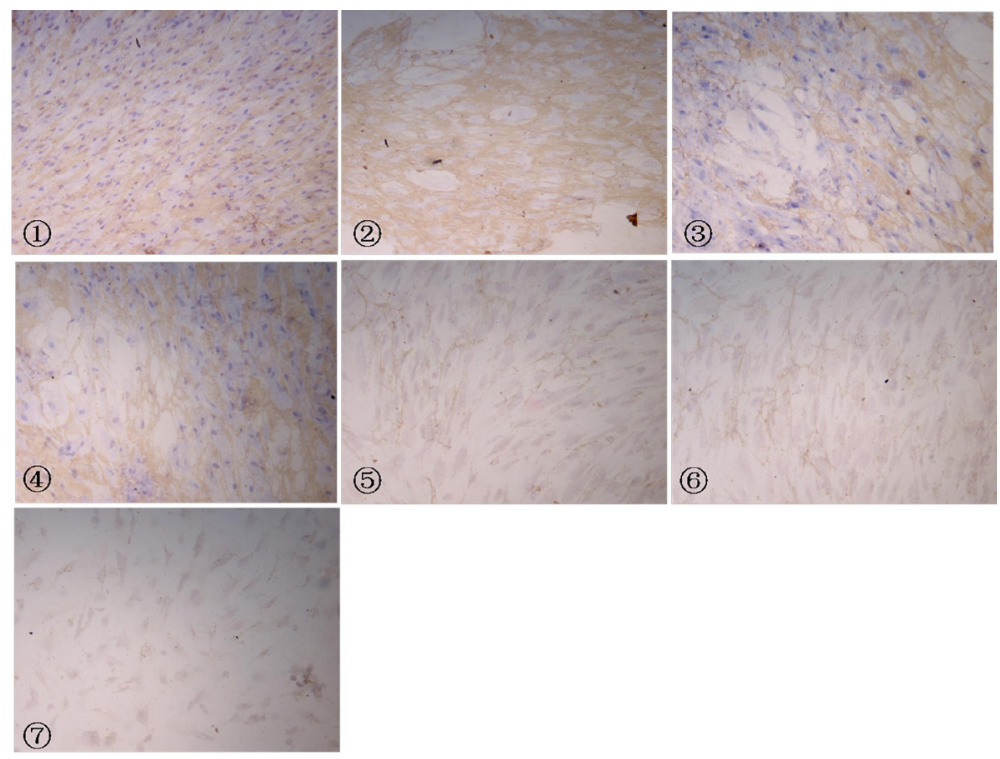

Figure 5. Effects of rifampicin at different concentrations on the expression of type collagen after 28 days of bone formation induction by immunochemistrial staining. Numbers $1,2,3,4,5,6$, and 7 represent $0,4,8,16,32,64$, and $128 \mu \mathrm{g} / \mathrm{mL}$ rifampicin treatment group, respectively. 

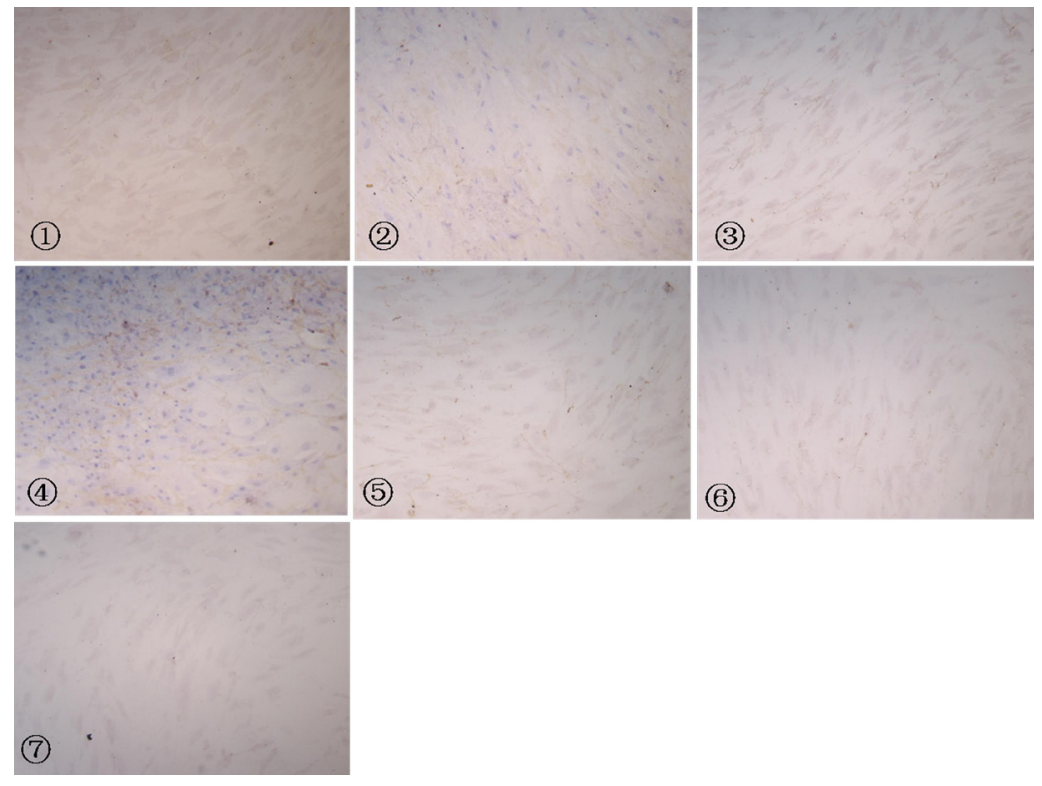

Figure 6. Effects of rifampicin at different concentrations on the expression of OPN/OPN/BGP after 28 day of bone formation induction by immunochemistrial staining. Numbers 1, 2, 3, 4, 5, 6, and 7 represent $0,4,8,16,32,64$, and $128 \mu \mathrm{g} / \mathrm{mL}$ rifampicin treatment group, respectively.

\section{Effects of rifampicin on OPN/BGP, COL1A1, and ALP expression by RT-PCR}

As shown in Table 3 and Figure 7, when the rifampicin concentration was $\geq 16 \mu \mathrm{g} / \mathrm{mL}$, the expressions of the OPN/BGP, COL1A1, and the ALP genes were significantly reduced compared with those of the control group $(\mathrm{P}<0.05)$.

Table 3. Gene expressions of RUNX2, COL1A1 and ALP determined by real-time PCR.

\begin{tabular}{lccc}
\hline Groups & & Gene relative expression & ALP/18S \\
\cline { 2 - 4 } & OPN/BGP /18S & COL1A1/18S & $0.093 \pm 0.001$ \\
1 & $0.617 \pm 0.002$ & $0.167 \pm 0.001$ & $0.076 \pm 0.001$ \\
2 & $0.510 \pm 0.001$ & $0.139 \pm 0.001$ & $0.063 \pm 0.002$ \\
3 & $0.490 \pm 0.002$ & $0.114 \pm 0.002$ & $0.053 \pm 0.001^{*}$ \\
4 & $0.450 \pm 0.002^{*}$ & $0.105 \pm 0.001^{*}$ & $0.046 \pm 0.002^{*}$ \\
5 & $0.347 \pm 0.001^{*}$ & $0.085 \pm 0.001^{*}$ & $0.037 \pm 0.001^{*}$ \\
6 & $0.221 \pm 0.001^{*}$ & $0.068 \pm 0.001^{*}$ & $0.023 \pm 0.001^{*}$ \\
7 & $0.121 \pm 0.002^{*}$ & & $0.045 \pm 002^{*}$ \\
\hline
\end{tabular}

*Compared with the control group, $\mathrm{P}<0.05$.

\section{Effects of rifampicin on RUNX2, COL1A1, and OPN/BGP expression by Western blot}

After 28 days of cell induction, the protein expressions of RUNX2, COL1, and OPN/ BGP were significantly decreased with increasing rifampicin concentration at concentrations $\geq 16 \mu \mathrm{g} / \mathrm{mL}$ compared with those of the control group $(\mathrm{P}<0.05, \mathrm{P}<0.01)$ (Figure 8). 
A

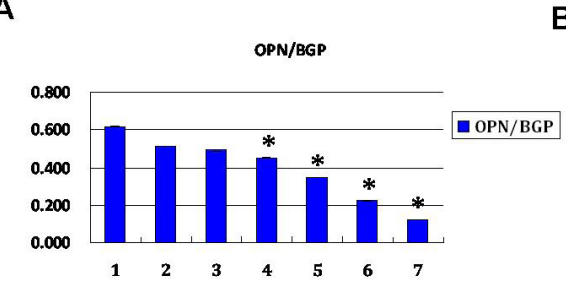

B

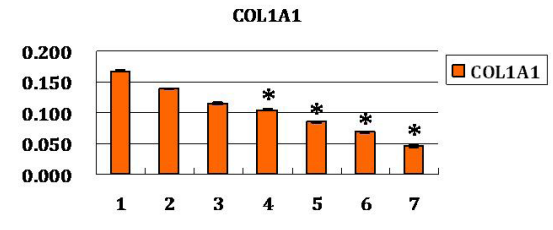

C

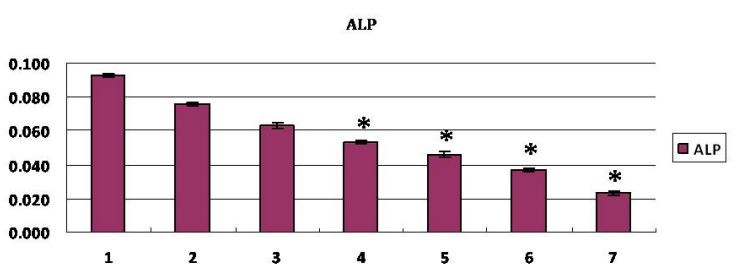

Figure 7. Effects of rifampicin at different concentrations on the expressions of bone formation genes in MSCs after 28 days of bone formation induction by real-time PCR. A. RUNX2. B. COL1A1. C. ALP.
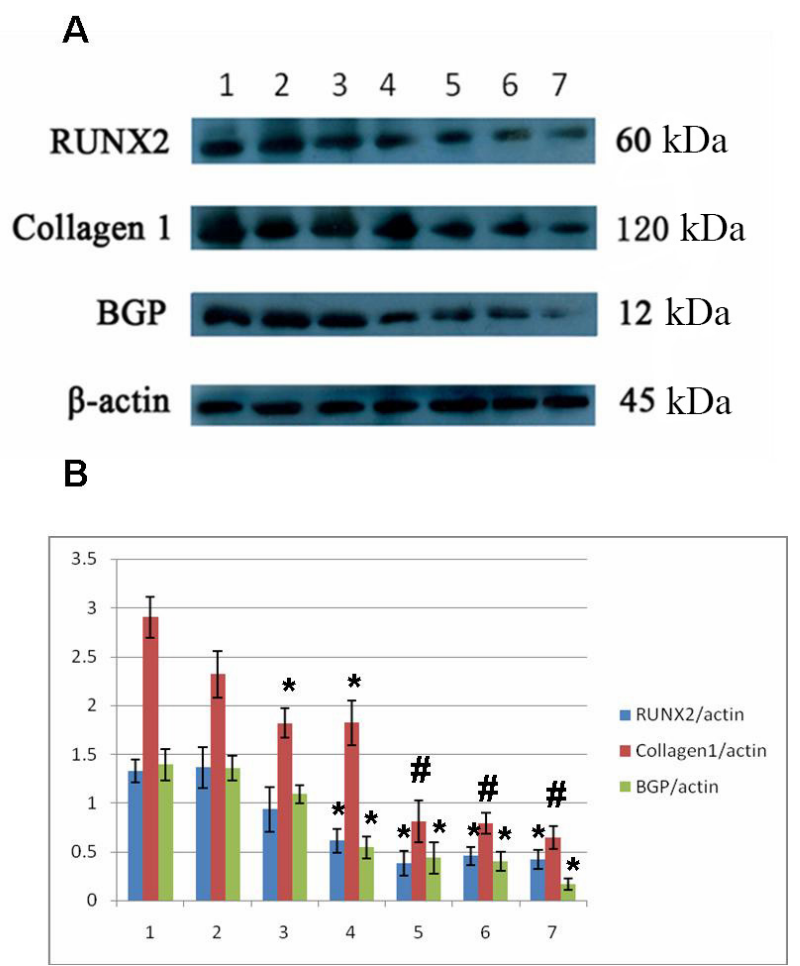

Figure 8. Effects of rifampicin at different concentrations on the expressions of bone formation proteins in MSCs after 28 days of bone formation induction by Western-blot. A. Result of RUNX2, Collagen 1 and OPN/OPN/BGP expressions detected by Western-blot. B. Column graph according to Western-blot analysis. Numbers 1-7 in long axis represent $0,4,8,16,32,64$, and $128 \mu \mathrm{g} / \mathrm{mL}$ rifampicin treatment group. ${ }^{*} \mathrm{P}<0.05$; ${ }^{*} \mathrm{P}<0.01$. 


\section{DISCUSSION}

Rifampicin is one of the most important drugs used in the treatment of tuberculosis, but has also recently been proven to be effective in the management of bone infections. Rifampicin is a potent antibiotic that is especially effective against Staphylococcus, which sometimes show resistance to beta-lactam antibiotics (Henry et al., 1987; Dworkin et al., 1990; O'Reilly et al., 1992; Littlewood-Evans et al., 1997; Rissing, 1997; Costerton et al., 1999). Furthermore, rifampicin has been shown to be an effective agent for treating bacteria in biofilms (Widmer et al., 1992), which Costerton et al. (1999) reported to be important in the pathogenesis of bone and joint infection. Therefore, rifampicin has been recommended in the treatment of prosthesis infections (Rissing, 1997). However, whether local high concentrations of antibiotics can affect local bone tissue and bone healing remains debatable. Therefore, this study was designed to investigate the effects of different rifampicin concentrations on the proliferation and differentiation of MSCs.

The main purpose of the local application of sustained-release drugs is to allow the target tissue to obtain the highest drug concentration possible while maintaining a low blood concentration of the drug to avoid systemic side effects. In order to evaluate whether the sustained-release system has value in clinical applications, this study was designed to adopt allogeneic bone and fibrin polymers as the drug stent of rifampicin, whose good biocompatibility has been widely confirmed. Therefore, we focused on the effects of different rifampicin concentrations on the proliferation and differentiation of MSCs in vitro. If rifampicin concentration is too high, it will produce cytotoxic effect and affect proliferation, transformation, and bone formation of MSCs. Therefore, the main objective of this study was to define the optimal concentration range of rifampicin that would not affect the activity of MSCs. Our results confirmed that $0-8 \mu \mathrm{g} / \mathrm{mL}$ rifampicin had no effect on inducing osteogenic differentiation of MSCs at the genetic level, whereas the osteogenic differentiation of MSCs was inhibited when the rifampicin concentration exceeded $16 \mu \mathrm{g} / \mathrm{mL}$.

The MTT assay can indirectly reflect the number of cells, and it can therefore be used to evaluate cell growth and proliferation. Our experimental results showed that with increased rifampicin concentration, the proliferation of MSCs was inhibited, particularly when the concentration exceeded $32 \mu \mathrm{g} / \mathrm{mL}$. The presence of rifampicin could induce MSC proliferation in each group; however, when the concentration of rifampicin was higher than $32 \mu \mathrm{g} / \mathrm{mL}$, cell proliferation reached a peak at the fifth day and then declined, which indicated that rifampicin at high concentrations caused cell necrosis and apoptosis. Furthermore, MSC microscopy showed that at high rifampicin concentrations, the cell bodies of the cells were swollen, and most cells had fallen off of the culture bottle.

During the process of endochondral ossification, RUNX2 is the earliest marker of bone formation, and can activate expressions of the ALP and OPN/BGP genes, as well as the productions of other bone-specific proteins and enzymes. The type I collagen level reflects the traction capacity of tissues, whereas ALP and OPN/BGP are mineralization-related proteins. Accordingly, we detected the activity of ALP and alizarin red-stained calcium nodules, and detected the relative quantitative gene expressions of ALP, OPN/BGP, and COLI. Our results suggested that increasing rifampicin concentration progressively inhibited the osteogenic differentiation of MSCs, particularly when the concentration of rifampicin was $\geq 16 \mu \mathrm{g} / \mathrm{mL}$.

Mature osteoblasts can secrete specific markers, such as osteocalcin and type I col- 
lagen, among others. Osteocalcin is a type of non-collagen protein secreted by osteoblasts, plays an important role in regulating bone calcium metabolism, and is a specific and sensitive biochemical marker for studying bone metabolism. In the present study, different rifampicin concentrations were combined with MSCs for bone induction culture, and its osteogenic effects were observed. The results of dynamic observations under inverted microscope showed that the cells in groups 1, 2, and 3 grew well, which secreted a high refraction of vacuoles and dark granules, and the cells around nodules were radially distributed. The cells in group 4 showed necrosis, and the cells in groups 5, 6, and 7 showed swelling, degeneration, and necrosis. There were significant differences in the morphology of cells in groups 4 to 7 compared with those in groups 1,2, and 3. The degree of this difference was enhanced with increasing rifampicin concentration. In addition, the results further confirmed that concentrations of $0-8$ $\mu \mathrm{g} / \mathrm{mL}$ rifampicin had no effect on inducing osteogenic differentiation of MSCs at the genetic level, whereas osteogenic differentiation of MSCs was inhibited when the rifampicin concentration was higher than $16 \mu \mathrm{g} / \mathrm{mL}$.

In conclusion, high rifampicin concentrations exert adverse effects on the proliferation of MSCs in human bone marrow and the differentiation of osteoblasts, particularly at concentrations higher than $16 \mu \mathrm{g} / \mathrm{mL}$.

\section{Conflicts of interest}

The authors declare no conflict of interest.

\section{REFERENCES}

Costerton JW, Stewart PS and Greenberg EP (1999). Bacterial biofilms: a common cause of persistent infections. Science 284: $1318-1322$.

Dworkin R, Modin G, Kunz S, Rich R, et al. (1990). Comparative efficacies of ciprofloxacin, pefloxacin, and vancomycin in combination with rifampin in a rat model of methicillin-resistant Staphylococcus aureus chronic osteomyelitis. Antimicrob. Agents Chemother. 34: 1014-1016.

Henry NK, Rouse MS, Whitesell AL, McConnell ME, et al. (1987). Treatment of methicillin-resistant Staphylococcus aureus experimental osteomyelitis with ciprofloxacin or vancomycin alone or in combination with rifampin. Am. J. Med. 82: 73-75.

Isefuku S, Joyner CJ and Simpson AH (2001). Toxic effect of rifampicin on human osteoblast-like cells. J. Orthop. Res. 19: 950-954.

Littlewood-Evans AJ, Hattenberger M, Zak O and O'Reilly T (1997). Effect of combination therapy of rifampicin and azithromycin on TNF levels during a rat model of chronic osteomyelitis. J. Antimicrob. Chemother. 39: 493-498.

Mahajan M, Rohatgi D, Talwar V, Patni SK, et al. (1997). Serum and cerebrospinal fluid concentrations of rifampicin at two dose levels in children with tuberculous meningitis. J. Commun. Dis. 29: 269-274.

O’Reilly T, Kunz S, Sande E, Zak O, et al. (1992). Relationship between antibiotic concentration in bone and efficacy of treatment of staphylococcal osteomyelitis in rats: azithromycin compared with clindamycin and rifampin. Antimicrob. Agents Chemother. 36: 2693-2697.

Rissing JP (1997). Antimicrobial therapy for chronic osteomyelitis in adults: role of the quinolones. Clin. Infect. Dis. 25: $1327-1333$.

Roth B (1984). Penetration of parenterally administered rifampicin into bone tissue. Chemotherapy 30: 358-365.

Widmer AF, Gaechter A, Ochsner PE and Zimmerli W (1992). Antimicrobial treatment of orthopedic implant-related infections with rifampin combinations. Clin. Infect. Dis. 14: 1251-1253.

Zimmerli W, Widmer AF, Blatter M, Frei R, et al. (1998). Role of rifampin for treatment of orthopedic implant-related staphylococcal infections: a randomized controlled trial. Foreign-Body Infection (FBI) Study Group. JAMA 279: $1537-1541$. 